\title{
COROT - A Unique Database for Low Amplitude Variability Between 1 Minute and 150 Days
}

\author{
E. Michel ${ }^{1}$, A. Baglin ${ }^{2}$, P. Barge ${ }^{3}$, C. Catala ${ }^{4}$, M. Auvergne ${ }^{1}$, W. W. \\ Weiss $^{5}$, T. Appourchaux ${ }^{6}$, R. Garrido ${ }^{7}$, and the COROT Team \\ ${ }^{1}$ Observatoire de Paris, DASGAL, UMR CNRS 8633, Meudon, France \\ ${ }^{2}$ Observatoire de Paris, DESPA, URA CNRS 264, Meudon, France \\ ${ }^{3}$ Laboratoire d'Astronomie Spatiale, CNRS, Marseille, France \\ ${ }^{4}$ Observatoire Midi-Pyrénées, LAT, UMR 5572, Toulouse, France \\ ${ }^{5}$ Institute of Astronomy, Vienna University, Wien, Austria \\ ${ }^{6}$ ESA/ESTEC, Noordwijk, the Netherlands \\ ${ }^{7}$ Instituto de Astrofisica de Andalucia, Granada, Spain
}

\begin{abstract}
COROT is a high precision wide field photometry experiment from space, funded in the framework of the CNES "Petites Missions" program (the PI is A. Baglin). It will observe approximately 30000 objects with $m_{V}$ between 4.5 and 15.5 over long observational periods (up to $150 \mathrm{~d}$ ), with a time sampling between $1 \mathrm{~s}$ and $16 \mathrm{~min}$, a precision of the order of $10^{-4}$ per measurement. The scientific objectives are stellar seismology and the search for telluric planets. The instrument and the core program have already been presented in several places. We here focus on a description of the characteristics of the data to be obtained with COROT. The large amount of high quality data collected by COROT will constitute rich material for several research programs beyond the core program as already defined (http://www.astrsp-mrs.fr/www/corot.html). A call for proposal of additional programs will be made during Northern Spring 2000.
\end{abstract}

\section{COROT - Mission Profile}

COROT is designed to run for at least 3 years, during which two observational programs will be followed:

-The Central Program: 5 to 6 long runs ( $150 \mathrm{~d}$ each). Each of these runs will be dedicated to a given field on the sky $\left(2.8^{\circ} \times 2.8^{\circ}\right)$, selected according to its stellar content. Considering the objectives of the stellar seismology program, these runs are mainly intended to obtain high precision $(0.1 \mu \mathrm{Hz})$ measurements of the oscillation frequencies in order to make possible a precise investigation of the stellar interiors. Considering the objectives of the exoplanet program, these runs are intended to bring about 10 telluric planets and a representative sample. of "hot Jupiters" for planetology investigations. 
-The Exploratory Program: 10 to 20 runs (10-20 d each). Each of these runs will be dedicated to a given field on the sky $\left(2.8^{\circ} \times 2.8^{\circ}\right)$. These runs are mainly devoted to the exploration of the pulsational behavior of stellar objects between spectral type $B$ and $K$.

During each run:

-Half of the field is designed to perform the observation of 5 to 10 objects with $m_{V}$ between 4.5 and 9 and with a time sampling of $1 \mathrm{~s}$.

-The second half of the field is designed for observing 6000 objects with $m_{V}$ between 12 and 15.5, with chromatic information (schematically a two-band measurement) for 2000 of them. The regular time sampling is $16 \mathrm{~min}$, but it will be possible to have 5 -min sampling for up to 35 objects in each field.

Table 1. Main characteristics of the observational outcomes

\begin{tabular}{ccccc}
\hline \multicolumn{6}{c}{5 to 6 runs of $150 \mathrm{~d}$ each, plus 10 to 20 runs of $10-20 \mathrm{~d}$ each. } \\
For each run:
\end{tabular}

*These numbers result from the scientific specification for periods smaller than a few hours (typically $\nu>70 \mu \mathrm{Hz}$ ).

**in the Fourier spectrum. $5 \mathrm{~d}$ being taken as a representative lifetime for solar-like oscillations in the stars considered here.

\section{Call for Additional Programs}

The large amount of high quality photometric data collected by COROT (see Table 1) will constitute rich material for several research programs beyond the core program of stellar seismology and the search for exoplanets. A call for proposal of additional programs will be made during Northern Spring 2000.

Possibilities for what can be allowed in an additional program are: addressing scientific questions not included in the core program, either using the same data, or asking for different targets and/or for different procedures than the core program; this may be in the same field of view (i.e. asking to open a new CCDwindow for a given object in the selected field), or in a different field (within the instrumental possibilities).

Additional programs will be selected by the COROT Scientific Committee by early 2001, and the proposers will become Guest Investigators. 